$20 \mid 2015$

NOVECENTO... E DINTORNI

Foscolo e la cultura europea

\title{
Foscolo en français : approche et critique des traductions
}

Foscolo in francese: approccio e critica delle traduzioni

Foscolo in French: Approach and Criticism of Translations

\section{Sarah Béarelle}

\section{OpenEdition}

\section{Journals}

Édition électronique

URL : http://journals.openedition.org/cei/2488

DOI : $10.4000 /$ cei.2488

ISSN : 2260-779X

\section{Éditeur}

UGA Éditions/Université Grenoble Alpes

Édition imprimée

Date de publication : 30 juin 2015

Pagination : 171-188

ISBN : 978-2-84310-289-9

ISSN : 1770-9571

\section{Référence électronique}

Sarah Béarelle, « Foscolo en français : approche et critique des traductions », Cahiers d'études

italiennes [En ligne], 20 | 2015, mis en ligne le 01 janvier 2017, consulté le 27 mars 2021. URL : http:// journals.openedition.org/cei/2488; DOI : https://doi.org/10.4000/cei.2488 


\title{
FOSCOLO EN FRANÇAIS : \\ APPROCHE ET CRITIQUE DES TRADUCTIONS
}

\author{
Sarah Béarelle \\ Université libre de Bruxelles
}

Explorer la postérité d'un auteur, jauger sa réception, c'est-à-dire mesurer son succès et son influence, consiste à articuler des informations chiffrables, quantifiables - comme le nombre d'éditions, de traductions, d'articles critiques, etc. - et des données qualitatives qui relèvent d'un domaine plus friable, labile et discutable, dans la mesure où il dépend de l'appréciation du lecteur, celui de l'influence de l'œuvre d'un écrivain sur un autre.

Nous aborderons Ugo Foscolo à travers le prisme des traductions françaises qu'il a générées et ce, grâce à une analyse essentiellement macrologique de celles-ci. Cette contribution aurait pu porter le titre de «Foscolo et ses réécrivains français». Formulation qui s'appuie sur les propos du traductologue Jean-René Ladmiral, lequel définit le traducteur comme un "coauteur", un "réécrivain ${ }^{\mathrm{I}}$ en tant qu'il passe le texte original au crible de sa subjectivité. Subjectivité qui frappe deux fois : en premier lieu, à la réception de l'œuvre et, dans un second temps, dans la phase d'écriture, de production, de "re-production». Cette prise en considération de l'empreinte de subjectivisme de toute traduction corrobore la formule tautologique mais qui a souvent fait défaut à l'histoire littéraire : le texte de la traduction n'est pas l'original ${ }^{2}$. Telle est la caractéristique épistémologique de la traduction qui sous-tend nos recherches. Mais asseoir notre approche sur les traductions de Foscolo requiert une prospection préalable des traductions françaises, démarche qui en appelle à l'histoire des textes

I. J.-R. Ladmiral, Traduire : théorèmes pour la traduction, Paris, Gallimard, 20IO, p. II2.

2. "Objection préjudicielle», pour reprendre G. Mounin, que ce dernier explicite dans cette formule inaugurale de son essai sur Les belles infidèles: «Toutes les objections contre la traduction se résument en une seule : elle n'est pas l'original.» (Id., Les belles infidèles, Lille, Presses Universitaires de Lille, I994 [1955].) 
et à leur pérennisation par l'acte traductif. Ainsi, notre étude se fonde avant tout sur une approche bibliométrique, nécessaire en amont d'une analyse traductologique inhérente à celle de la réception d'un auteur.

Nous entendons, dans un premier temps, tracer le parcours emprunté par la traduction française des œuvres foscoliennes et en montrer les sillages afin de dégager l'image de Foscolo qui transparaît dans cette opération de transfert d'une langue à l'autre. Pour ce faire, notre corpus considère les différents aspects de la production scripturale de l'auteur, à la fois romancier, poète, dramaturge et critique. Concernant le 'Foscolo poète', nous avons circonscrit notre enquête à deux ouvres emblématiques : I Sepolcri et Le Grazie. Concernant les autres domaines de sa plume, nous avons inventorié et collationné toutes les traductions intégrales présentant des versions distinctes. En outre, nous avons distingué les traductions partielles des intégrales, les traductions de texte et la traduction de l'œuvre. Parmi cet ensemble, seront dans un premier temps laissées hors champ les traductions de texte, qui relèvent de la version - dont le modèle serait l'exercice scolaire homonyme - pour nous focaliser sur celles de l'œuvre. Dans un second temps, nous approcherons l'œuvre ayant eu, jusqu'à ce jour, le plus de prégnance, la pénétration la plus significative, et nous analyserons le schéma dynamique de ses traductions françaises. Sans prétendre à l'exhaustivité vu la pluralité complexe des relations extrinsèques auxquelles sont soumises les traductions, notre intention est de faire ressortir, par la voie diachronique, un certain nombre de tendances, de déterminer les périodes où la retraduction est active, de formuler des hypothèses sur les liens de causalité du phénomène traductionnel, et de dégager des pistes sur les statuts de la production foscolienne suite à son passage au français.

Mais aborder de manière bibliométrique les traductions de Foscolo, en faire l'archéologie ${ }^{3}$, considérer celles-ci comme des sources documentaires, n'est réalisable que consécutivement à un travail minutieux de fouilles en vue d'établir la bibliographie la plus exhaustive qui soit - si tant est que l'on puisse prétendre à l'exhaustivité dans le domaine bibliographique car l'on sait que tout travail de ce type suppose en effet un système de références ouvert et non définitif. Afin de ratisser au plus large et de répertorier des versions françaises qui auraient échappé aux bibliographes de

3. Concept du traductologue A. Pym : la «translation archeology is a set of discourses concerned with answering all or part of the complex question "who translated what, how, where, when, for whom and with what effect?" It can include anything from the compiling of catalogues to the carrying out of biographical research on translators" (A. Pym, Method in Translation History, Manchester, St. Jerome Publishing, I998, p. 6). 
Foscolo, ont été retenues, d'une part, les traductions déposées en France et indexées dans le Catalogue collectif de France ainsi que celles qui, tout en étant publiées à l'étranger, ont fait l'objet d'acquisitions par les bibliothèques ou institutions enregistrées dans celui-ci et, d'autre part, celles renseignées en Italie dans le Catalogo del servizio bibliotecario nazionale. D'autres encore ont été dévoilées au fil de nos lectures et grâce à l'exploitation et à l'exploration de documents critiques. C'est généralement par ce modus operandi que nous avons rencontré les publications dans des revues et découvert les entreprises de réécrivains qui n'ont pas eu de relief dans les anthologies de Foscolo. Après ces considérations heuristiques, auscultons ce que nous disent les traductions françaises de l'ensemble des œuvres foscoliennes.

\section{Foscolo et ses traductions}

\section{Foscolo romancier}

Dans une perspective diachronique, observons ce qu'il en est des Ultime lettere di Jacopo Ortis et du Sesto tomo dell'Io (fig. I). Un premier constat saute aux yeux : la présence dominante de l'Ortis face à l'unique traduction $\mathrm{du}$ Sesto tomo dell'Io de Michel Orcel en $1984^{4}$. L'on dénombre dix versions françaises différentes' de l'Ortis dont plusieurs proviennent du même traducteur, ce qui donne lieu à une succession de textes, à une véritable chaîne de traductions dont chaque maillon participe au processus d'intégration de l'œuvre dans la culture française. Il est pertinent de noter la coexistence dans la translatio de l'Ortis d'une tradition manuscrite — l'on relève trois

\footnotetext{
4. U. Foscolo, Le sixième tome du moi, traduit et commenté par M. Orcel, Paris, L'Alphée, I984.

5. Les ouvrages suivants présentent une version du texte à chaque fois originale; les traductions issues du même traducteur répertoriées ici témoignent d'un retravail du texte et/ou du titre : U. Foscolo, Dernières lettres de Jacques Ortis, librement traduites de l'italien sur la $3^{\mathrm{e}}$ édition, par H.-A. Passerat de la Chapelle, ms. 66, Paris, Médiathèque Élisabeth et Roger Vailland, I8ı3; U. Foscolo, Le proscrit, ou Lettres de Jacopo Ortis, traduites de l'italien sur la $2^{\mathrm{e}}$ édition par M. de S[enonnes], 2 vol., Paris, Pillet, I8I4; U. Foscolo, Les dernières lettres de Jacopo Ortis, imprimées sur les manuscrits autographes, traduites de l'italien [par A. Trognon], Paris, Delestre-Boulage, I8I9; U. Foscolo, Amour et suicide, le Werther de Venise, [par de Senonnes], 2 vol., Paris, Dentu, I820; U. Foscolo, Dernières lettres de Jacopo Ortis, traduites sur l'édition de Milan, I824, par PhélippesBeaulieu, ms 740, Nantes, Médiathèque Jacques Demy, ı824; U. Foscolo, Jacopo Ortis, traduit de l'italien par M. G[osselin], traducteur des Fiancés, 2 vol., Paris, Dauthereau, I829; A. Dumas, Jacques Ortis, Paris, Dumont, I839; A. Dumas, Jacques Ortis, précédé d'un essai sur la vie et les écrits d'Ugo Foscolo, par Eugène de Montlaur, et suivi d'une traduction inédite de ses Euvres choisies, par L. Delâtre, Paris, C. Gosselin, I842; U. Foscolo, Dernières lettres de Jacques Ortis. 4 octobre 1797, Bibliothèque municipale de Tournus, ms. 90, I852; U. Foscolo, Les dernières lettres de Jacopo Ortis, roman traduit de l'italien par J. Luchaire, Paris, Société française d'imprimerie et de librairie, 1906.
} 


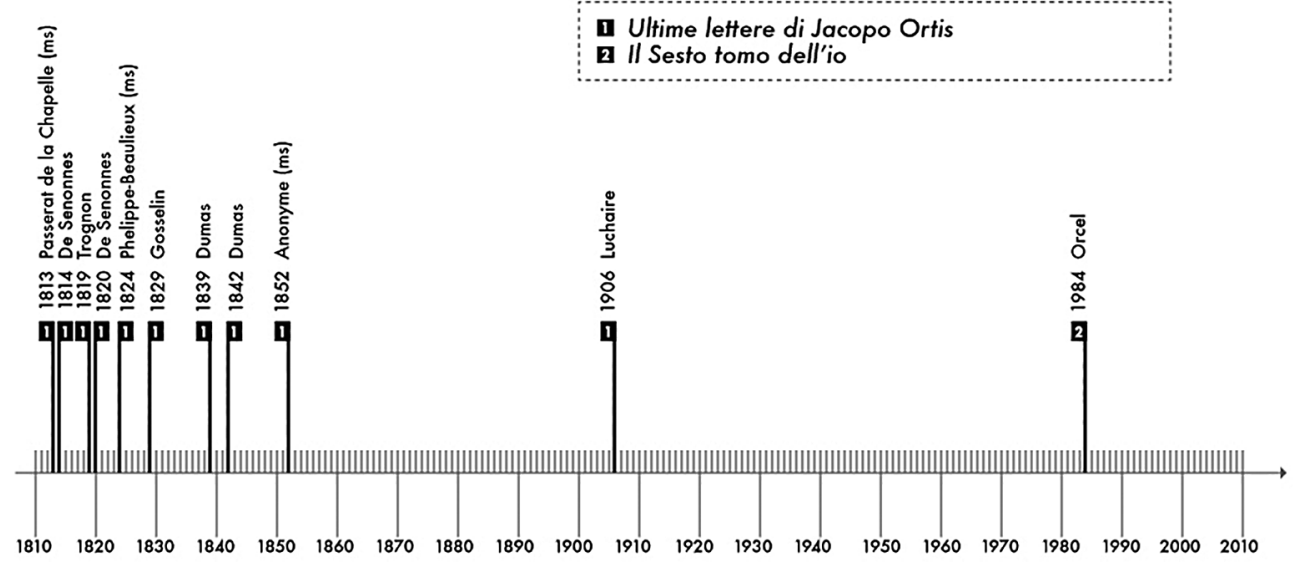

Fig. I. - Foscolo romancier.

manuscrits - et d'une transmission d'imprimés. Selon les logiques du phénomène de retraduction, l'on peut affirmer que puisque retraduction du roman il y a, de même il y a canonisation ou du moins reconnaissance de l'œuvre. Du point de vue chronologique, l'Ortis s'installe en France entre I8I3 à 1906, et plus significativement dans la première moitié du xIx ${ }^{\mathrm{e}}$ siècle.

\section{Foscolo critique}

Si l'on observe le Foscolo critique (fig. 2), l'on remarque une infime pénétration de ses écrits dans le domaine français, dont les premières incursions témoignent toutefois d'une grande réactivité des traducteurs vu le court laps de temps qui sépare la publication de la traduction et l'émission de l'original. Ces textes critiques paraissent dans des revues - la Revue européenne ${ }^{6}$, la Revue britannique ${ }^{7}$ — dont la politique éditoriale était, dans une plus ou moins large mesure, de présenter en traduction des textes provenant d'autres périodiques européens. Louis Delâtre fut le premier à soumettre un écrit critique de Foscolo en dehors de cette logique éditoriale des revues mais non sans l'influence d'une stratégie de publication puisque

6. U. Foscolo, «Principes de critique poétique appliqués plus particuliérement à la langue italienne», Revue européenne, I, août I824, p. 24I-252; Id, "Première époque - Depuis l'année II80 jusqu'en I230", Revue européenne, I, octobre I824, p. 534-555; Id., "Deuxième époque - Depuis I230 jusqu'à I280", Revue européenne, II, novembre I824, p. 78-94; Id., "Origine et vicissitudes de la langue italienne», Revue européenne, I, septembre I824, p. 339-348.

7. U. Foscolo, "Constitution démocratique de Venise (Edinburgh Review)", Revue britannique, juillet I827, p. 282-314. 


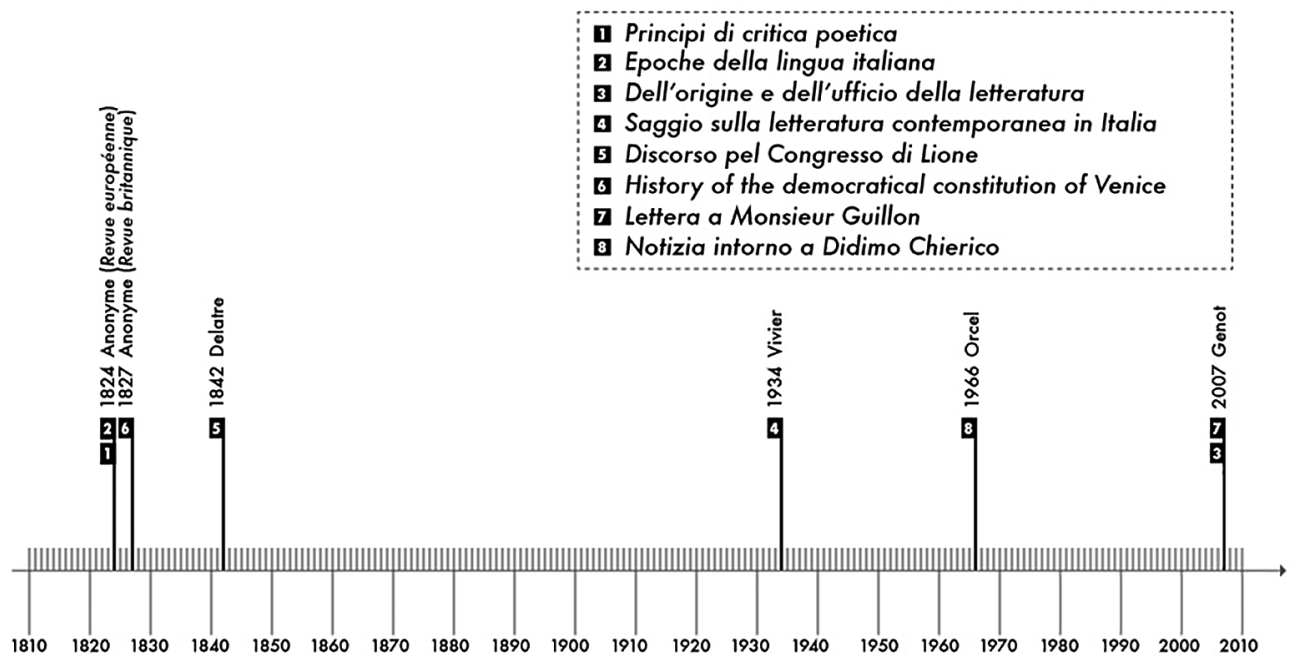

Fig. 2. - Foscolo critique.

sa traduction du Discorso pel Congresso di Lione paraît en guise de paratexte de la traduction de l'Ortis d'Alexandre Dumas père ${ }^{8}$. Il est alors légitime de penser que cette traduction s'inscrit dans une logique commerciale qui contribue à étayer l'image de l'auteur italien. Dans ce même esprit, la traduction complète du Saggio sulla letteratura contemporanea in Italia s'insère dans un volume anthologique charpenté selon les différentes facettes de l'œuvre de Foscolo — y compris le 'Foscolo épistolier' — regroupant des traductions intégrales et fragmentaires de l'italianiste Robert Vivier ${ }^{9}$. En 1966, Michel Orcel fournit au lectorat français la Notizia intorno a Didimo Chierico ${ }^{\mathrm{IO}}$ et, en 2007, près de deux siècles après l'original, Gérard Genot sort de l'ombre Dell'origine e dell'ufficio della letteratura ${ }^{\mathrm{II}}$ et la Lettera a Monsieur Guillon. S'il est manifeste qu'un 'Foscolo critique' existe du point de vue traductif dans le domaine français, il n'y a pas, à proprement parler, de réelle pénétration ni de reconnaissance de celui-ci tant il a été peu traduit intégralement, surtout si l'on considère qu'aucune retraduction ne vient participer à une pérennisation de ces écrits.

8. U. Foscolo, Discours à Bonaparte au Congrès de Lyon, trad. L. Delâtre, dans A. Dumas, Jacques Ortis, précédé d'un essai, ouvr. cité, p. 223-255.

9. U. Foscolo, Essai sur l'état de la littérature italienne, dans R. Vivier, Ugo Foscolo. Poésies, proses choisies, Paris, La Renaissance du Livre, «Les cent chefs-d'œuvre étrangers», I934, p. 235-25I.

IO. U. Foscolo, "Notizia intorno a Didimo Chierico». Notice sur Didyme Leclerc, introduction et traduction de G. Genot, Paris, Lettres modernes, 1966.

II. U. Foscolo, "De l'origine et des devoirs de la littérature", suivi de "Les tombeaux" et "Les sonnets»; introduction, traduction et notes de G. Genot, Lausanne, Paris, L’Âge d'Homme, 2007. 


\section{Foscolo dramaturge}

Attardons-nous un moment sur le Foscolo dramaturge (fig. 3) : une seule traduction témoigne de ce versant de l'œuvre foscolienne, celle de la Ricciarda ${ }^{12}$. L'historien, écrivain et professeur Auguste Trognon s'insère dans le projet éditorial de la collection «Chefs-d'œuvre des théâtres étrangers» dirigée par Ladvocat. Par conséquent, même si, d'un point de vue chronologique, cette traduction témoigne d'un rapide temps de réaction $\mathrm{du}$ traducteur puisque celui-ci donne une version française deux ans après la publication de la pièce chez John Murray, l'on ne peut pas dire que Foscolo s'impose en France par son ouvre dramatique ${ }^{\mathrm{I} 3}$.

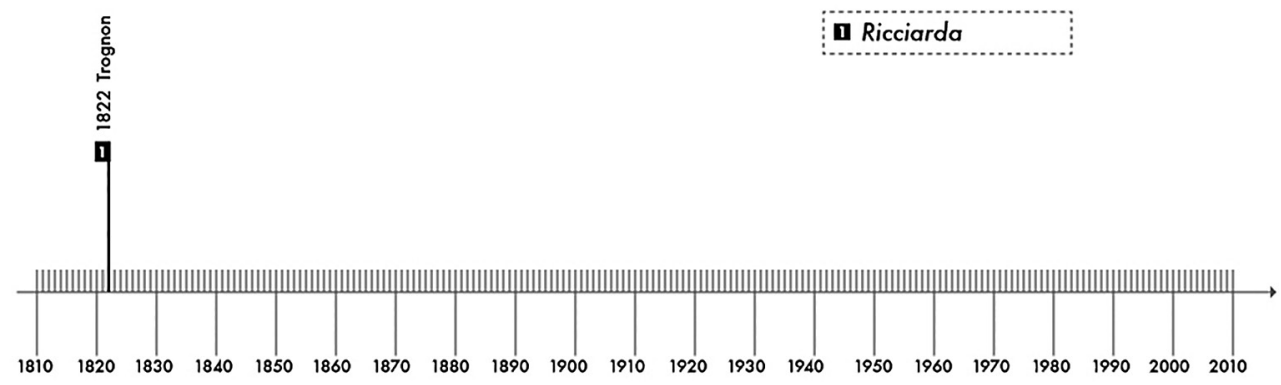

Fig. 3. - Foscolo dramaturge.

\section{Foscolo poète}

La fortune du 'Foscolo poète', observée à travers I Sepolcri et Le Grazie, se déploie bien différemment (fig. 4). Force est de constater que les Sepolcri ont une plus nette et incontestable pénétration que les Grazie dont on ne comptabilise que deux occurrences, l'une dans l'ouvrage anthologique du sus-évoqué Robert Vivier, Ugo Foscolo. Poésies et proses choisies, l'autre dans une anthologie poétique également consacrée à Foscolo mais avec le texte original en vis-à-vis, L'ultime déesse, élaborée par Michel Orcel. L’on recense par contre quatorze versions françaises différentes des Sepolcri ${ }^{i 4}$

I2. U. Foscolo, Ricciarda, trad. A. Trognon, dans A. Trognon (éd.), Chefs-d'ceuvre du théâtre italien moderne, t. I, Paris, Ladvocat, "Chefs-d'œuvre des théâtres étrangers", I822, p. 99-I67.

13. Sur la fortune française de la Ricciarda, nous renvoyons à la contribution de Paola Ranzini dans le présent volume.

I4. U. Foscolo, Les Tombeaux, trad. L. Delâtre, dans A. Dumas, Jacques Ortis, précédé d'un essai, ouvr. cité, p. 257-266; G. Chatenet, "Les Tombeaux, traduction en vers", dans Id., Étude sur les poètes italiens, Paris, Fischbacher, 1892; A. Fighiera, Ugo Foscolo. Poésies, première traduction française avec le texte en regard, un 


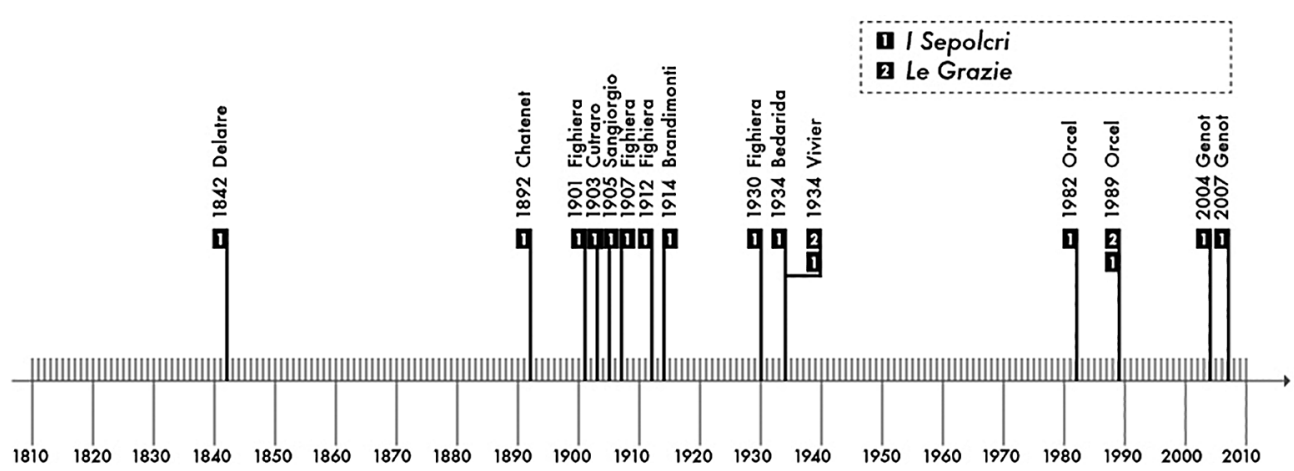

Fig. 4. - Foscolo poète.

pour dix traducteurs. En effet, pour I Sepolcri, les rééditions vont toujours de pair avec une révision du texte de la part du traducteur et donnent lieu à un nouvel état du texte ${ }^{15}$. L'arrivée du poème est lente, puisque la publication de l'original date de 1807 et que Louis Delâtre inaugure le Foscolo des Sepolcri en français en 1842 , en guise d'apparat à la traduction dumasienne de l'Ortis. Et il n'y aura pas d'autre traducteur avant la toute fin du $\mathrm{xIx}^{\mathrm{e}}$ siècle, où l'on assiste à une recrudescence de retraductions initiée par Gustave Chatenet dans son Étude sur les poètes italiens en I892. Les suivantes sont des traductions publiées en Italie. Lorsque, dans les écrits français, l'on voit poindre la première monographie française consacrée à Foscolo ${ }^{16}, D e i$ Sepolcri sont alors repris par des professeurs universitaires et italianistes : Henri Bédarida publie une "nouvelle traduction» dans la revue Dante et Robert Vivier dans son anthologie foscolienne. Michel Orcel et Gérard Genot reviendront plus récemment sur le poème. Le premier, en 1982, propose une traduction dans un recueil intitulé Les Tombeaux et autres poésies, traduction qu'il retravaille pour la publication de son anthologie foscolienne L'ultime déesse; le second publie sa version en 2004, dans la revue Chroniques italiennes, avant de la joindre, sous une forme remaniée, à la publication de sa traduction de Dell'origine e dell'ufficio della letteratura.

notice biographique et des notes, Savone, Bertolotto, I907 (I9I2, 1930 et même I90I); B. Cutraro, Essai de traduction en vers "Les sépulcres» de Hugues Foscolo, Trévise, Istituto Turazza, 1903; G. Sangiorgio, I Sepolcri, tradotti in prosa francese, dans Id., Feuilles de laurier, Faenza, Novelli e Castellani, s. a. [1905]; Les Tombeaux, traduits par S. Brandimonti, Messine, Guerriera, I9I4; H. Bédarida, «Les Tombeaux, ode», Dante, janvier 1934; R. Vivier, Ugo Foscolo, ouvr. cité, p. I4I-I5O; U. Foscolo, Les tombeaux et autres poèmes, traduits et présentés par M. Orcel, Rome, Académie de France, "Villa Médicis», I982; U. Foscolo, L'ultime déesse, Paris, La Différence, 1989, p. 60-83; G. Genot, "Traduction de I Sepolcri de Ugo Foscolo», Chroniques italiennes, 73/74, 2004/2-3, p. 47-6o; U. Foscolo, De l'origine, ouvr. cité.

15. Tel est le cas pour les travaux traductifs de Fighiera, Orcel et Genot.

I6. A. Caraccio, Ugo Foscolo. L'homme et le poète, Paris, Hachette, I934. 
S'il est évident que toutes ces observations sont à recouper avec les différentes composantes d'une étude de réception étayée - le contexte historique, socio-culturel, commercial, etc. - pour cerner la fortune de Foscolo en France, les modélisations typologiques des traductions que nous avons élaborées permettent déjà par elles-mêmes de dégager des tendances. Bien entendu, il n'est pas licite de corréler la valeur littéraire d'une œuvre ou encore son succès commercial au nombre de ses retraductions, mais établir pareille archéologie permet toutefois de cerner partiellement son insertion dans une culture autre. Ce panorama de Foscolo en français nous indique notamment les œuvres ayant connu la présence la plus forte et la plus durable sur le territoire français. Il s'agit donc des Sepolcri, avec quatorze versions françaises intégrales pour dix traducteurs et, en second lieu, de Ultime lettere di Jacopo Ortis dont on dénombre dix versions intégrales différentes pour neuf traducteurs. Mais si, comme nous l'évoquions, les rééditions des Sepolcri appelaient à un retravail du texte de la part du traducteur, les rééditions ou même les réémissions de l'Ortis sont à considérer dans le cas où l'on veut réellement cerner les moyens par lesquels le public français pouvait connaître l'œuvre. Si l'on ajoute donc aux éditions originales de l'Ortis français leurs rééditions ou leurs réémissions (fig. 5), les périodisations se modifient : d'une phase de traduction qui s'étalait de I8I3 à 1906, on passe désormais à un intervalle de temps plus large qui atteste de la présence de l'œuvre jusqu'en I994. 'Foscolo poète' cède ainsi le pas au 'Foscolo romancier' et à son Jacopo puisque de dix versions intégrales françaises, l'on passe à dix-neuf Ortis.

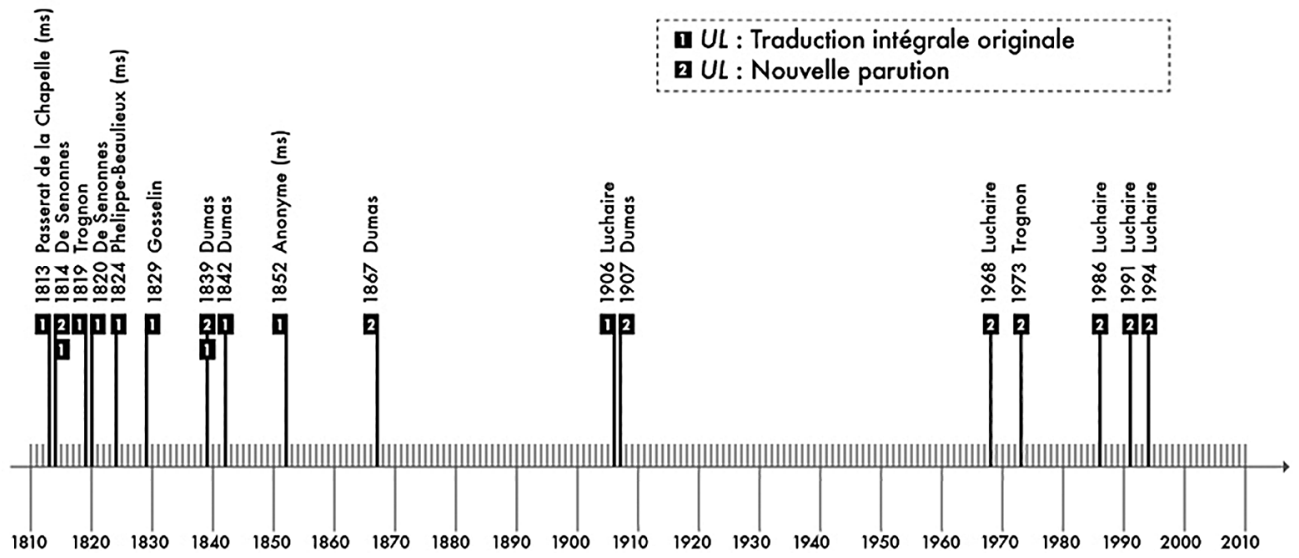

Fig. 5. - L'Ortis, traductions intégrales. 


\section{L'Ortis et ses traductions}

Afin d'exploiter au maximum l'approche bibliométrique des traductions et de compléter l'historiographie de l'Ortis en français, nous inclurons dans notre analyse les traductions partielles (fig. 6). Cette vue diachronique de la chaîne traductive de l'Ortis, avec tous ses maillons, nous permet de revenir sur l'histoire du texte original qui, comme on le sait, a connu des péripéties dans son parcours éditorial et présente une véritable stratigraphie. Établir une archéologie des traductions de l'Ortis consiste à remonter aux sources de celles-ci. Pour ce faire, la correspondance de Foscolo est un matériel de première main qui enrichit l'histoire du traduire de l'auteur et lui donne plus de profondeur.

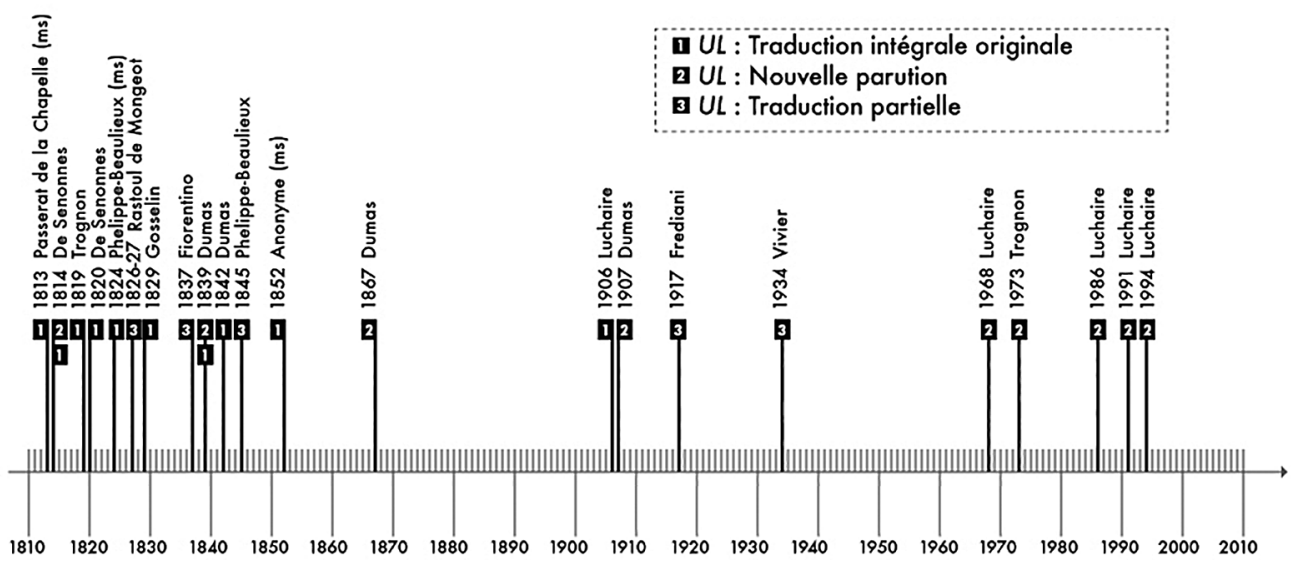

Fig. 6. - L'Ortis, traductions intégrales et partielles.

La première allusion à une traduction française de l'Ortis trouve sa source dans une lettre que Foscolo envoie de Florence le 2 janvier I8or aux éditeurs de la Gazzetta universale dans laquelle il rejette la Vera storia di due amanti infelici. Édition, selon Foscolo, qui en a engendré d'autres parmi lesquelles il renseigne, par prétérition : "Tacerò di una versione francese stampata dagli Alains a Parigi e pubblicata da pochi mesi a Milano. Tutto è al solito refondu, corrigé et augmentét ${ }^{17}$ ". Il s'agirait donc d'une traduction du premier état du texte. Angelo Ottolini, dans sa Bibliografia foscoliana, mentionne une "traduzione in francese delle Ultime lettere» publiée à 
Paris par Alcains ${ }^{18}$, mais cette référence ne sera plus jamais renseignée par les bibliographes et demeure énigmatique. En effet, bien que l'auteur fasse état de l'existence de cette traduction aucune trace de celle-ci ne nous est parvenue; et il en va de même pour la maison d'édition — qu'il s'agisse de Alains ou Alcains. Cette traduction reste inconnue et son existence ne peut être affirmée.

Durant le séjour en France de Foscolo au sein de la division italienne, nous retrouvons des évocations de projets traductifs. Dans une lettre que lui adresse en septembre I805 Maurice Guibourg, cet expéditeur se rappelle au bon souvenir de l'auteur italien en se qualifiant de "traducteur de l'Ortis qui va faire tout son possible pour se procurer un exemplaire de votre ouvrage qu'il aime autant que son auteur ${ }^{19}{ }^{\prime}$. En outre, la même année, dans sa correspondance avec Amélie Bagien, Foscolo déclare vouloir lui-même traduire les Ultime lettere ${ }^{20}$. Il semblerait par ailleurs qu'une certaine Madame Lenormant ${ }^{21}$ entreprit de traduire le roman en français, mais cette dame nous reste inconnue tout autant que sa présumée tentative de traduction. Toutes ces déclarations ne sont restées qu'ambition et n'ont jamais atteint le stade de la concrétisation. Si elles sont des traductions fantômes, elles témoignent toutefois de l'engouement que suscitait alors le roman et attestent de la volonté même de Foscolo de favoriser sa propre diffusion en français.

Dans son numéro du 7 février i8II, le Journal de l'Empire prévient ses lecteurs de la parution prochaine de la traduction des Ultime lettere chez l'éditeur Dentu. Deux jours plus tard, la Gazette de France en annonce une chez l'éditeur Lefèvre. Mais ces annonces furent alors classées sans suite puisque le roman, quelques mois auparavant, avait été mis sous séquestre par la politique napoléonienne en vigueur qui reprochait au roman de «représenter la domination française [...] comme une insupportable tyrannie et [d']exciter tous les peuples qui y sont soumis au soulèvement et à la révolte ${ }^{22} »$.

Mais cette censure, si elle réprima la publication de traductions, n'étouffa pas le désir de traduire de certains. Ainsi, le comte GeorgesHonoré-Anthelme Passerat de la Chapelle (I779-I865), qui fut officier

18. A. Ottolini, Bibliografia foscoliana: contenente la descrizione di tutte le opere di Ugo Foscolo e delle traduzioni delle stesse opere la rassegna cronologica degli studi riguardanti il Foscolo tre indici accuratissimi per materia per nomi e per riviste, Florence, Battistelli, I92I, p. II.

19. Ep. II, p. 72.

20. Ep. II, p. $75-76$.

2I. Ep. II, p. 90-92.

22. Le rapport est conservé aux Archives nationales de France (AF, IV, I354); cf. G. Bourgin, «Le Ultime lettere di Jacopo Ortis et la censure impériale", Études italiennes, I919, p. 229. 
durant la première campagne d'Italie ${ }^{23}$, signe la première traduction française du roman ${ }^{24}$. Cette traduction est restée à l'état de manuscrit et est conservée dans le fonds patrimonial de la Médiathèque de Bourg-enBresse. Le traducteur ambitionnait initialement de soumettre son texte au public mais le conseiller d'État de la direction générale de l'imprimerie lui refusa ce projet ${ }^{25}$.

C'est à De Senonnes, pseudonyme d'Alexandre de la Motte-Baracée $\left(\mathrm{I} 78 \mathrm{I}-\mathrm{I} 84 \mathrm{O}^{26}\right)$ que l'on doit la première publication dans le domaine français de l'Ortis en version française ${ }^{27}$, en I8I4, après l'abdication de Bonaparte. Cet admirateur de l'Italie, écrivain, ardent royaliste, souligne dans son avertissement le sort jusqu'alors réservé au roman : «Faut-il ajouter maintenant que cette traduction fut arrêtée par ordre supérieur, il y a quelques années, au moment de paraître?» et rappelle le sort identique qui lui fut réservé dans son pays en précisant qu' «aujourd'hui, peutêtre, une semblable persécution devient un avantage». Avantage dans sa diffusion qui semble poursuivre l'œuvre et déterminer sa portée puisque De Senonnes dans sa péroraison éclaire son projet traductif et son travail dans une sorte de conquestio puisque "on ne saurait du moins s'empêcher de rendre quelque justice à l'énergie d'un homme qui osa, dans ces jours de servitude, faire entendre des paroles de liberté, et signaler courageusement à la haine publique l'oppresseur de sa patrie ${ }^{28} »$. Et cette intention, De Senonnes la marque également dans le titre : Ultime lettere di Jacopo Ortis deviennent Le proscrit ou Lettres de Jacopo Ortis. D'exilé italien, le protagoniste passe au rang d'immigré français. Ce texte sortira la même année avec les mêmes caractéristiques physiques, chez l'éditeur Lefèvre ${ }^{29}$. Foscolo eut vent de cette traduction et il l'évoque dans le chapitre de la Notizia bibliografica intitulé «Traduzioni ${ }^{30}$ ». Dans cette partie, il est question de plusieurs versions du roman en langue étrangère : la traduction allemande

23. Sur Passerat de la Chapelle, voir entre autres : E. Révérend du Mesnil, Armorial historique de l'Ain, Bresse, Bugey, Dombes, pays de Gex, Valromey et Franc-Lyonnais, Lyon, A. Vingtrinier, I872, p. 496; C.-J. Dufaÿ, Dictionnaire biographique des personnages notables du département de l'Ain. Galerie militaire de l'Ain, depuis les temps les plus reculés jusqu'à nos jours, Bourg, L. Grandin, 1874, ad vocem.

24. Voir plus haut, n. 5 .

25. La lettre du conseiller d'état est reproduite par Passerat de la Chapelle dans son manuscrit.

26. Sur ce dernier, nous revoyons à : [s. n], "Alexandre de La Motte-Baracée», dans L.-G. Michaud, Biographie universelle ancienne et moderne : histoire par ordre alphabétique de la vie publique et privée de tous les hommes avec la collaboration de plus de 300 savants et littérateurs français ou étrangers, Paris, Desplayes, I843-1865 ( ${ }^{\mathrm{e}}$ éd.), t. XXXIX, p. 80-8I.

27. U. Foscolo, Le proscrit, ouvr. cité.

28. Ibid., p. IV.

29. U. Foscolo, Le proscrit, ou Lettres de Jacopo Ortis, trad. de l'italien sur la $2^{e}$ éd. par M. de S[enonnes], Paris, Lefève, 2 vol., I8I4.

30. EN IV, p. $483-485$ 
par Luden en 1807 , la traduction anglaise et la traduction française publiées toutes deux en I8I4. À leur égard, Foscolo formule un reproche inhérent à l'acte de traduire, toutes «smentiscono la fama dell'originale». Mais tandis que les travaux traductifs anglais et allemand optent pour une grande littéralité, la traduction de De Senonnes «è caduta nel contrario e peggiore difetto ». Le texte en français est complètement modifié, dénaturé même : "per abbreviarlo talvolta, allargarlo spesso, e abbellirlo sempre, lo trasfigura in guisa che taluni leggendola hanno sospettato che fosse traduzione della Vera Storia di due amanti infelici pubblicata da Angiolo Sassoli, anziché delle Lettere di Jacopo Ortis ${ }^{3 \mathrm{I}}$. Foscolo aiguise encore sa critique envers cette traduction :

Senza che, molti passi sono traintesi, molti travolti a bella posta; e il senso vien a rovescio dell'originale: il che s'incontra principalmente ne' ragionamenti concatenati con rapida e stretta brevità, ne' quali chi tradusse non ha sottinteso i nodi tralasciati dall'autore. Finalmente il traduttore non si contentò nè del frontespizio nè d'un volumetto solo: lo divise in due e lo chiamò Le proscrit ${ }^{32}$.

Pour notre auteur, son roman ne peut être lisible dans d'autres idiomes et moins encore en français, pourtant langue sœur : «è presso che impossibile il tradurlo in francese, idioma che ha per indole la chiarezza e l'esattezza, e sopra tutto certa elegenza di convenzione e di bon ton, due cose ignote agli scrittori originali delle altre nazioni ${ }^{33}$ ».

Pourtant, d'autres s'aventurèrent dans cette entreprise frôlant l'impossible. En I8I9, Auguste Trognon donne une nouvelle version de l'Ortis milanais auquel il restitue le titre original ${ }^{34}$. Dans son avertissement, le traducteur rappelle au lecteur que le livre «devint une puissance assez redoutable pour provoquer les persécutions du despotisme ombrageux qui pesait alors sur la République cispalpine ${ }^{35}$ »; il insiste sur le fait que ces «quelques déclamations contre la tyrannie et les tyrans ne pouvaient fournir le cadre d'un ouvrage pleinement intéressant ${ }^{36}{ }^{\prime}$. Le seul réel intérêt que l'on puisse y trouver est, selon le traducteur, le récit amoureux. Pour Trognon, il n'y a pas d'intrigue et nuance-t-il, "c'est à peu de chose près l'action du roman de Werther, transportée en Italie, au milieu des orages

3I. Ibid., p. 484 .

32. Ibid.

33. Ibid., p. 484-485. À propos des considérations foscoliennes sur les traductions dans la Notizia bibliografica, cf. G. Nicoletti, Ancora sulla «Notizia» zurighese, dans S. Bearelle, L' "Ortis» e la Francia. Approcci e prospettive, Bruxelles, Peter Lang, 20I4, p. 39-47.

34. U. Foscolo, Les dernières lettres, traduites par A. Trognon, ouvr. cité.

35. Ibid., p. 8.

36. Ibid., p. 8-9. 
révolutionnaires ${ }^{37}$ ». C'est là le référent qu'il donne au public français afin qu'il cerne au mieux le texte qui va suivre. Un «Voyez Werther, vous cernerez l'Ortis" qui semble renvoyer à la mode de l'époque en France, si l'on en juge le nouveau titre sous lequel De Senonnes reprend sa traduction en 1820 : les Ultime lettere di Jacopo Ortis sont alors publiées sous le nom de Le Werther de Venise ${ }^{38}$.

La collation des traductions permet d'observer que la censure pèse encore sur l'Ortis car, si l'on s'attend, en I8I9 et en I820, à une actualisation par souci d'adhérence philologique au nouvel état et même nouveaux états du texte, c'est-à-dire à la version zurichoise (I8I6) de l'Ortis et à celle de Londres (I8I7), c'est celle de I802 que le traduire fait circuler. L'Ortis avait été réélaboré et une actualisation dans le domaine des traductions s'imposait mais les traducteurs semblaient frileux. Attitude à laquelle le crible de la censure ne devait pas être étranger.

C'est par le biais d'un manuscrit, une fois encore, que l'on peut découvrir la première version française de l'Ortis zurichois ${ }^{39}$. Se basant sur l'édition des Ultime lettere parue à Milan en $1824^{40}$, un jeune avocat nantais, Emmanuel Phélippes-Beaulieu, donne la première traduction française de l'Ortis de 1816 et va même au-delà puisque, par une comparaison de son édition originale et d'une édition de $\mathrm{I} 8 \mathrm{O} 2$, il relève les passages qu'il dit «retranchés par acte du Pouvoir». Cette déclaration témoigne que le traducteur ne connaissait pas l'histoire des différentes versions de l'original. Phélippes-Beaulieu reviendra sur son manuscrit dix ans plus tard mais il ne sera jamais publié dans son intégralité ${ }^{4}$.

En I826, l'Ortis de I8I6 s'infiltre dans le domaine public via les pages du journal lyonnais L'indépendant ${ }^{42}$. Son directeur, Alphonse Rastoul de Mongeot y traduit sept lettres dans sept livraisons du journal. Il traduit les lettres datées du 20 novembre, 28 octobre, 3 décembre, 22 novembre, I4 mai et I4 mai au soir 1798 et celle du I4 mai I799. Il présente donc une sorte d'Ersatz d'Ortis que Lionello Sozzi, dans son article consacré à la littérature italienne dans la presse lyonnaise, verra comme une volonté

37. Ibid., p. 9.

38. U. Foscolo, Amour et suicide, ouvr. cité.

39. U. Foscolo, Dernières lettres, par Phélippes-Beaulieu, ouvr. cité.

40. U. Foscolo, Ultime lettere di Jacopo Ortis tratte dagli autografi, Milano, appresso T. Barrois \& Jombert libraj, e si trova in Parigi, I824.

4I. U. Foscolo, Dernières lettres de Jacopo Ortis, fragments d'une traduction inédite par M. PhélippesBeaulieu, Nantes, Mellinet, I845.

42. A. Rastoul de Mongeot, Lindépendant, journal de Lyon, des départements, de Paris, et de l'étranger, 7 lettres: $\mathrm{n}^{\text {os }} \mathrm{I} 8,20,36,43,46,48$, II9, I826-I827. 
de Rastoul de Mongeot de mettre en avant le pétrarquisme du roman foscolien ${ }^{43}$.

Mais le premier traducteur à introduire la version intégrale de l'Ortis zurichois par sa traduction française est Pierre-Joseph Gosselin ${ }^{44}$. Il se pose volontairement en opposition par rapport à ses prédécesseurs dont les traductions sont pour lui "fautives» car, précise-t-il, c'était un Ortis mutilé qui circulait précédemment ${ }^{45}$.

Le Napolitain Pier Angelo Fiorentino ${ }^{46}$ traduit également l'Ortis zurichois mais en feuilleton dans le journal parisien La Presse ${ }^{47}$. Dans un article présentant Foscolo, il annonce sa traduction et son horizon comme suit : "Pour montrer à nos lecteurs la ressemblance frappante qui existe entre l'œuvre et l'auteur, entre l'original et la copie, nous n'avons qu'à citer quelques fragments des Dernières lettres ${ }^{48}$ ». Ces fragments ont donc pour but de décrire l'auteur dans un jeu spéculaire avec son personnage et pour ce faire, Fiorentino traduit partiellement la lettre de Padoue, celle du 4 décembre à Milan et celle du 19 et 20 février écrite à Ventimiglia. Mais ces coupes opérées dans le roman lui attribuent un autre skopos et l'insèrent dans une autre logique.

Pier Angelo Fiorentino revient sur l'Ortis en I839 mais, cette fois, en qualité de préfacier puisque c'est comme protégé d'Alexandre Dumas père qu'il rédige la préface du Jacques Ortis de ce dernier. Ainsi, une grande figure de la littérature française s'adonne à la traduction de l'Ortis zurichois $^{49}$, livre avec lequel Dumas avoue avoir appris l'italien ${ }^{50}$. Avec ce Jacques Ortis, Dumas participe à la pérennisation du roman jusqu'au début $\mathrm{du} \mathrm{xx}^{\mathrm{e}}$ siècle. Mais il sera alors proposé au lecteur français dans les collections consacrées aux œuvres de Dumas ${ }^{\text {II }}$.

43. L. Sozzi, «La letteratura italiana nella stampa lionese degli anni I8I5-I848», Études italiennes, I936, p. 43. 44. U. Foscolo, Jacopo Ortis, traduit de l'italien par M. G[osselin], traducteur des Fiancés, Paris, Dauthereau, 2 vol., I829.

45. Ibid., p. 5 .

46. Sur ce dernier, voir entre autres: G. Monsagrati, «Fiorentino, Pier Angelo», dans Dizionario biografico italiano, Rome, Istituto della enciclopedia italiana, vol. 48, 1997, p. I57-I60; C. F. Goffis, "Fiorentino, Pier Angelo", dans Enciclopedia dantesca, Rome, Istituto dell'Enciclopedia Italiana, vol. II, I970, p. 902.

47. P. A. Fiorentino, "Variétés. L'art en Italie. Ugo Foscolo», La Presse, 26 septembre 1837 et Id., «Feuilleton de la presse. L'art en Italie. Ugo Foscolo. II", La Presse, I5 décembre I837.

48. Id., "Variétés", art. cité.

49. A. Dumas, Jacques Ortis, Bruxelles, Meline, Cans et C., I839 et Id., Jacques Ortis, Paris, Dumont, I839.

50. «Un des livres dans lesquels j'ai appris l'italien était le beau roman d'Ugo Foscolo, que j'ai traduit depuis sous le titre de Dernières lettres de Jacopo Ortis.» (A. Dumas, Mes Mémoires, choix et présentation par G. Giraux, Paris, Union générale d'éditions, 1962, t. I, p. 138.)

5I. Sur l'Ortis dumasien, nous renvoyons à notre étude : S. Béarelle, "L'Ortis di Alexandre Dumas», dans Id. (éd.), L' «Ortis» e la Francia, ouvr. cité, p. I09-ı20. 
De I839 à la fin du xix ${ }^{\mathrm{e}}$ siècle, c'est cette récupération de l'Ortis par Dumas qui va assurer sa diffusion car, si l'on voit poindre d'autres résurgences du roman, il s'agit de traductions partielles et d'exercices pédagogiques qui relèvent d'un désir personnel de se livrer à la tâche du traduire. En effet, en I845, à Nantes, l'auteur du manuscrit de la première version française de l'Ortis zurichois, Emmanuel Phélippes-Beaulieu, fait imprimer quelques passages retravaillés de son texte auxquels il joint un avertissement expressément écrit pour cette publication dans lequel il invective ses prédécesseurs et expose son projet traductif ${ }^{52}$. Ce qu'il souhaite, c'est «élever, sur le sol de la France, un monument au souvenir d'un enfant des Hellènes, à ce jeune homme dont l'existence orageuse a présenté des ressemblances avec la vie du chantre d'Armide ${ }^{53}$ ». "Erigere un monumento alla virtù sconosciuta», formule du personnage de Lorenzo dans l'Ortis, fait écho à son projet traductif. Toutefois les six lettres, avec rupture chronologique, ainsi que le fragment pour Lauretta qu'il traduit, sont bien peu pour présenter le roman.

À la bibliothèque de Tournus, sommeille un manuscrit anonyme d'une traduction de l'Ortis. Il s'agit d'une version de l'Ortis milanais, un retour au premier texte donc. Elle est couchée sur un cahier et l'absence de tout commentaire du traducteur laisse à penser que l'entreprise a été purement et simplement un exercice linguistique et pédagogique.

Pour revenir à l'Ortis de Dumas, d'un point de vue macrostructurel, celui-ci sera présenté dans un premier temps en monade, en œuvre unique, pour ensuite être étoffé de renseignements sur la vie et les écrits de l'auteur italien par Eugène de Montlaur et de "pièces choisies" foscoliennes traduites par Louis Delâtres ${ }^{54}$ dans une volonté nette de souligner l'authenticité de l'original. Le texte sera ensuite récupéré dans des éditions destinées à présenter les écrits de Dumas, et le traducteur prendra le pas sur l'auteur, l'Ortis devenant un objet commercialisé dans des ouvres dumasiennes, dans des ouvrages entourés d'autres productions de Dumas 55 . Il passe dans le domaine des éditions à moindre coût de Michel Levy et s'insère même dans la collection "Alexandre Dumas illustré» de Le Vasseur en $1907^{56}$, livre d'illustrations présentant des gravures de Gustave Doré, Foulquier et Gerlier.

52. U. Foscolo, Dernières lettres de Jacopo Ortis, fragments d'une traduction inédite par M. PhélippesBeaulieu, Nantes, Mellinet, I845.

53. Ibid., p. 5 .

54. A. Dumas, Jacques Ortis, précédé d'un essai, ouvr. cité.

55. A. Dumas, Jacques Ortis. Les fous du docteur Maraglia, Paris, Michel Levy, 1867.

56. A. Dumas, Le maître d'armes, Jacques Ortis, La route de Varennes, Napoléon, Murat, Paris, Le Vasseur, 1907. 
En 1906, Ultime lettere di Jacopo Ortis réapparaît dans une «nouvelle traduction" de l'italianiste Julien Luchaire qui, le premier, traduit l'Ortis londonien. Il s'agit de la version qui perdure encore de nos jours. Dans son avertissement, Luchaire émet l'avis suivant sur le roman qu'il s'apprête à traduire :

[...] les Dernières Lettres sont un livre tout à fait immoral, — ou plutôt, pis que cela : démoralisant. Dans la catégorie des mauvais livres, il mériterait même une mention spéciale. Car il ne contient rien qui blesse les oreilles, et le grand amour d'Ortis pour Thérèse est chastement exprimé. C'est un livre à donner aux jeunes filles... qu'on ferait peut-être mieux de ne pas donner aux jeunes gens, du moins à certains. Ce romancier, qui n'est pas un philosophe de profession et ne cherche pas midi à quatorze heures, énonce, avec beaucoup d'éloquence mais beaucoup de vraisemblance aussi, et on dirait presque de bon sens, quelques-unes des meilleures raisons qui sont de désespérer de la vie ${ }^{57}$.

Le traducteur avance à pas feutrés et classe le roman dans la catégorie morale des «mauvais livres». S’il s'attelle à ce "vieux chef-d'œuvre» — ce sont ses mots —, sa volonté de «faire œuvre» n'est pas revendiquée ni même énoncée.

Joséphine Frediani donne un petit livret de quelques pages se présentant comme un "Essai de traduction» qui est basé sur une sélection de quelques lettres ${ }^{58}$. Dans un préambule à ces morceaux choisis, elle témoigne de l'admiration qu'elle a toujours éprouvée pour Foscolo et ses lettres. Dans son essai, elle fait coexister lettres réelles et lettres fictives en traduisant tant des passages du roman que des morceaux de la correspondance foscolienne.

L'Ortis sera traduit par Robert Vivier en 1934 pour son volume anthologique Ugo Foscolo, poésie, proses choisies. Si Vivier opte pour la version de l'Ortis zurichois, il en élimine des passages, dont la lettre du i7 mars.

Dans la deuxième moitié $\mathrm{du} \mathrm{xx}^{\mathrm{e}}$ siècle, en 1968, Georges Haldas reprend la version de Julien Luchaire et l'insère dans sa collection consacrée aux "Grandes heures de la littérature italienne» dans un volume où il traite, aux côtés de Foscolo, de Leopardi et de Mazzini ${ }^{59}$. En I973, l'Ortis d'Auguste Trognon, celui de I802 est réintroduit par Valeria Tasca dans la collection Helgé ${ }^{60}$. Cette collection, mise en place par René Guise, avait

57. U. Foscolo, Les dernières lettres, traduit par J. Luchaire, I906, ouvr. cité, p. XLIII.

58. J. Frediani, Essai de traduction, Massa, Medici, I9I7.

59. U. Foscolo, Les dernières lettres de Jacopo Ortis, trad. J. Luchaire, dans Grandes heures de la littérature italienne, sous la direction de G. Haldas, t. VII (Foscolo, Leopardi, Mazzini), Lausanne, Rencontre, 1968.

6o. U. Foscolo, Les dernières lettres de Jacopo Ortis, trad. de l'italien par A. Trognon, suivi de Notice bibliographique sur "Les dernières lettres de Jacopo Ortis» par U. Foscolo, trad. par V. Tasca; textes réunis, présentés et annotés par V. Tasca, Paris, Éd. du Delta, 1973. 
comme programme original des rééditions commentées de traductions anciennes. Ce projet didactique avait pour but de faire prendre conscience au lecteur de sa lecture en traduction, de lui proposer un véritable dossier sur le texte qu'il allait découvrir, portant tant sur l'original que sur sa traduction. L'Ortis est en réalité alors récupéré dans un projet didactique où le souci de l'éditeur était d'éclairer le lectorat sur l'actualisation de l'acte de lecture d'une traduction et sur le pacte traductologique entre le lecteur et l'œuvre.

Enfin, quatre-vingts ans après la dernière réécriture de l'Ortis ${ }^{6 \mathrm{I}}$, la maison d'édition toulousaine Ombres réédite la traduction de Luchaire, l'Ortis londonien. Sur la quatrième de couverture, l'intrigue est présentée comme suit :

Octobre 1797, Jacopo, désespéré de voir Venise sacrifiée par Bonaparte, se réfugie dans les Collines Euganéennes, près de Padoue. Il y rencontre Teresa; les fiançailles de la jeune fille interdisent une passion partagée. Commence alors un pèlerinage mélancolique à travers l'Italie; la douleur amoureuse s'y mêle à la véhémence du proscrit. Après le mariage de Teresa, Jacopo rejoint les collines et met fin à ses jours.

La composante amoureuse devient, pour l'édition française, le fer de lance du roman ; lecture qui se renforce par l'image que l'on trouve en couverture de cette édition : il s'agit d'une photographie anonyme (vers I850), représentant une jeune femme. Si l'on sait combien l'image d'entrée, ces "images d'exposition» jouent le rôle de "fenêtre» sur le roman, de «réclame» sur le texte qui va suivre, il ne nous semble pas erroné de postuler cette transformation de l'Ortis en roman dit «sentimental». Il en va de même pour la réédition que l'éditeur en donne en I99I, qui présente de manière identique le texte. La réédition qui nous est la plus proche, l'Ortis qu'il nous est donné de lire en français aujourd'hui, date de 1994. Si le texte de Luchaire demeure tel quel, les caractéristiques éditoriales se modifient : le format se réduit et Les dernières lettres de Jacopo Ortis font partie de la «Petite bibliothèque Ombres». La quatrième de couverture est revue et la publicité du roman amoureux est supprimée pour donner la part belle à l'auteur de «l'autobiographie [qui deviendra] le cri du Risorgimento ". L'accent est alors posé sur le lien auteur-personnage : "Son obsession est la Révolution française, son idéal l'Italie, son rêve la poésie, sa vie l'errance, la littérature, l’amour, le désespoir et toujours la lucidité violente d'un poète guerrier.» Le portrait de couverture passe au

6r. U. Foscolo, Les dernières lettres de Jacopo Ortis, roman traduit de l'italien par J. Luchaire, Toulouse, Ombres, 1986. 
masculin. C'est un autoportrait de Samuel Palmer (I826) qui, bien qu'il n'ait rien à voir avec l'iconographie foscolienne, colle avec la description physique que Foscolo faisait de lui dans l'Autoritratto.

\section{Conclusion}

Les différentes lignes de force qui ont été tracées pour qualifier l'image d'un "Foscolo français» s'érigent en une étude liminaire essentielle, un cadre nécessaire et préalable à tout examen de contenu. À partir de ce panorama de "Foscolo en français", il ressort que les œuvres de l'auteur ayant le plus suscité l'intérêt français sont I Sepolcri et Ultime lettere di Jacopo Ortis. D’un point de vue bibliométrique, eu égard aux traductions originales, partielles et reparutions, l'on observe que l'Ortis est l'œuvre foscolienne la plus ancrée dans le domaine français. En se focalisant sur les dynamiques de traduction et de retraduction, se sont esquissés les contours des différents statuts du roman dans sa réception française.

Dans une quasi-contemporanéité avec l'original, plusieurs témoignages attestent de l'enthousiasme d'un passage de l'œuvre en français. Mais la condamnation de l'Ortis sous l'Empire entrave les premières tentatives des traducteurs. Enfin, dès la Première Restauration, l'insertion de Ultime lettere di Jacopo Ortis est amorcée. Du statut de proscrit, d'une lecture déterminée par des considérations politiques, Jacopo passe, au fil de la multiplication des traductions, à celui de protagoniste d'une intrigue amoureuse, en avatar de Werther. Il rencontre la presse et, ensuite, la plume de Dumas. Lequel prendra le pas sur l'œuvre, l'Ortis se voyant alors diffusé comme élément des écrits dumasiens. Le roman foscolien se développe également en objet d'exercices formels, linguistiques et pédagogiques. Il prend aussi place dans des ouvrages d'érudition. Enfin, la version française intégrale la plus récente, la traduction de Luchaire datant de 1906, a connu un parcours en trois temps : de "vieux chef-d'œuvre» jugé selon des considérations morales, la version est ensuite reprise à la fin du $\mathrm{xx}^{\mathrm{e}}$ siècle avec, comme point de saillance, l'intrigue amoureuse; l'Ortis semble alors rattaché à la tradition du roman sentimental. Aujourd'hui, cette dominante amoureuse a cédé le pas à une assimilation Ortis-Foscolo, présentant le roman comme l'autobiographie d'un poète soldat, d'un vates guerrier. 